\title{
Philosophiques
}

\section{De Karl Bühler à Karl R. Popper}

\section{Fiorenza Toccafondi}

Volume 26, numéro 2, automne 1999

La critique de la raison en Europe centrale

URI : https://id.erudit.org/iderudit/004992ar

DOI : https://doi.org/10.7202/004992ar

Aller au sommaire du numéro

\section{Éditeur(s)}

Société de philosophie du Québec

ISSN

0316-2923 (imprimé)

1492-1391 (numérique)

Découvrir la revue

\section{Citer cet article}

Toccafondi, F. (1999). De Karl Bühler à Karl R. Popper. Philosophiques, 26(2), 279-300. https://doi.org/10.7202/004992ar

\section{Résumé de l'article}

Popper a passé sa licence sous la direction de Bühler en 1928. Affirmer que Popper a été profondément influencé, non seulement par la théorie du langage de Bühler, mais aussi par sa psychologie, ne correspond pas à l'opinion courante. Le deuxième chapitre de Die beiden Grundprobleme der Erkenntis montre clairement que Karl Bühler a représenté l'un des points de départ les plus importants de la théorie de l'esprit de Popper. Selon Popper, dans l'épistémologie de Carnap, il y a un " préjudice inductif » : afin de rétablir une opinion neutre et adopter une épistémologie qui soit indépendante de la psychologie, il est nécessaire de démontrer que, dans la psychologie aussi, le déductivisme est possible et concevable. Popper donne des exemples très clairs de psychologie déductive et cite la théorie de Kant, Johannes Mueller, l'École Wuerzburg (surtout à travers Bühler et Otto Selz) et Ernst Mach. Le choix de tels exemples est significatif afin de comprendre la nature de la formation de Popper. En particulier, il est important de souligner l'absence de la "psychologie de la Gestalt " ou de ses penseurs, tels que Wertheimer, Koehler, Koffka, etc. En effet, Bühler s'opposait fortement à la psychologie de la Gestalt de l'École Wertheimer et, entre l'Institut Bühler de Vienne et les psychologues de la Gestalt à Berlin, il y avait une véritable rivalité. Popper - qui est normalement considéré, de façon simpliste, comme étant « un psychologue gestaltiste » - a tout à fait adopté l'idée de Bühler.
Ce document est protégé par la loi sur le droit d'auteur. L’utilisation des services d’Érudit (y compris la reproduction) est assujettie à sa politique d'utilisation que vous pouvez consulter en ligne.

https://apropos.erudit.org/fr/usagers/politique-dutilisation/ 


\title{
De Karl Bühler à Karl R. Popper
}

\author{
FIORENZA TOCCAFONDI \\ Université de Florence \\ Fiorenzat@hotmail.com
}

RÉSUMÉ. - Popper a passé sa licence sous la direction de Bühler en 1928. Affirmer que Popper a été profondément influencé, non seulement par la théorie du langage de Bühler, mais aussi par sa psychologie, ne correspond pas à l'opinion courante. Le deuxième chapitre de Die beiden Grundprobleme der Erkenntis montre clairement que Karl Bühler a représenté l'un des points de départ les plus importants de la théorie de l'esprit de Popper. Selon Popper, dans l'épistémologie de Carnap, il y a un «préjudice inductif » : afin de rétablir une opinion neutre et adopter une épistémologie qui soit indépendante de la psychologie, il est nécessaire de démontrer que, dans la psychologie aussi, le déductivisme est possible et concevable. Popper donne des exemples très clairs de psychologie déductive et cite la théorie de Kant, Johannes Mueller, l'École Wuerzburg (surtout à travers Bühler et Otto Selz) et Ernst Mach. Le choix de tels exemples est significatif afin de comprendre la nature de la formation de Popper. En particulier, il est important de souligner l'absence de la «psychologie de la Gestalt » ou de ses penseurs, tels que Wertheimer, Koehler, Koffka, etc. En effet, Bühler s'opposait fortement à la psychologie de la Gestalt de l'École Wertheimer et, entre l'Institut Bühler de Vienne et les psychologues de la Gestalt à Berlin, il y avait une véritable rivalité. Popper — qui est normalement considéré, de façon simpliste, comme étant « un psychologue gestaltiste » - a tout à fait adopté l'idée de Bühler.

ABSTRACT. - Popper graduated under Bühler's direction in 1928. It's not part of the common view to say that Popper was strongly influenced, not only by Bühler's theory of language, but also by his psychology. The second chapter of Die beiden Grundprobleme der Erkenntnis shows clearly that one of the fundamental starting points for Popper's theory of mind was Karl Bühler. According to Popper, an "inductive prejudice" is contained in Carnap's epistemology : to restore a neutral point of view and to adopt an epistemology independent of psychology, it is necessary to show that, within the psychology, the deductivism is also possible, or thinkable. Popper provides some clear examples of deductive psychology and cites Kant's outlook, Johannes Mueller, the Wuerzburg's school (particularly by Bühler and Otto Selz) and Ernst Mach. The choice of such examples is very important for the understanding of the nature of Popper's training. It is significant, in particular, the absence of the Gestaltpsychology or of its exponents, such as Wertheimer, Koehler, Koffka etc. In fact, Bühler was in sharp contrast with the Gestaltpsychology of Wertheimer's school and between the Bühler's Vienna Institute and the Berlin Gestalt-psychologists there was a real rivalry. Popper - who by the standard view is simplistically considered "a gestaltist psychologist" - adopted completely the Bühler's point of view. 
1

Dans son Autobiographie, Popper propose une vaste rétrospective de sa formation intellectuelle ${ }^{1}$. Comparé à d'autres indications contenues dans certains de ses ouvrages antérieurs (en premier lieu dans Conjectures and R efutations et dans $\mathrm{O}$ bjective $\mathrm{K}$ nowledge), le compte-rendu de Popper est apparu « décevant », car le développement des problèmes et des idées de fond de son parcours de formation manque de « références au domaine relatif à la psychologie et à la philosophie de la science de cette période». Pour cette raison, certaines des « racines », des origines et certains des facteurs qui ont contribué à stimuler la pensée de Popper demeurent assez « vagues $»^{2}$; ainsi se heurte-t-on souvent à des auteurs qui introduisent sa pensée en la reliant directement à d'éminentes théories de l'histoire de la philosophie, en la présentant comme une formulation qui, « dans un certain sens, ne faisait que puiser, encore une fois, à la tradition de l'hypothético-déductivisme, dont [...] certains éléments remontent jusqu'à l'A ntiquité » ${ }^{3}$.

$C^{\prime}$ 'est cette situation qui a poussé à rechercher les « dettes » quePopper a confessées seulement de façon partielle. Par exemple, il y a plus d'une vingtaine d'années, W.W. Bartley III a déterminé la source de l'utilisation hypothético-déductiviste de la philosophie kantienne par Popper : Popper était un " psychologue gestaltiste», " à l'esprit façonné de manière décisive [par la pensée] de Bühler, de Külpe, et par celle des autres psychologues gestaltistes ${ }^{4}$. En Italie, dans la même lignée, certains ont proposé l'idée d'un Popper qui, « sous l'impulsion du gestaltisme, parvint à une position kantienne », et la thèse selon laquelle « le gestaltisme qui conduisait Popper au kantisme était l'antithèse de cet associationnisme psychologique qui, à l'inverse, menait à l'empirisme ou à l'idéal isme de Berkel ey et de M ach, qui fut typique des premières formulations néopositivistes $»^{5}$.

De l'interprétation de Bartley, on partage sans aucun doute l'idée que I'on doit rechercher les racines de la philosophie de Popper dans la formation psychologique menée sous la direction de $\mathrm{Karl}^{\mathrm{Büh}} \mathrm{er}^{6}$, ainsi que l'idée que cette période a exercé une influence beaucoup plus importante que celle explicitée par Popper. Pour être plus précis, l'influence exercée par Bühler

1. Cette rétrospective fait état de son travail à la clinique de $M$ ax A dler, des expéditions d'Eddington, de la lecture de Kant, Freud, M arx, C. Lord M organ et, enfin (mais de façon plus secondaire), de sa fréquentation du Psychologische Institut dirigé par Karl Bühler ; c'est à ces auteurs et à ces expériences en particulier que Popper attribue une grande importance dans le processus de sa formation intellectuelle (cf. Popper, 1974, p. 20, 35-36, 39, 42, 55, 62).

2. Wettersten, 1988, p. 327-329.

3. Oldroyd, 1986, p. 389.

4. Bartley, 1974, p. 13, 50-51.

5. Pera, 1981, p. 239.

6. En qualité d'étudiant, Popper commença à fréquenter Bühler vers le milieu des années vingt. C ette période marquera l'aboutissement de son mémoire ( $Z$ ur $M$ ethodenfrage der D enkpsychologie), dont la soutenance eut lieu en 1928 ; Bühler en fut le rapporteur, et M oritz Schlick, le corapporteur. 
devrait être expliquée non seulement (commec'est déjà le cas) par rapport au fait que « beaucoup d'idées de Popper apparemment révolutionnaires sont des applications directes de la philosophie de la psychologie évolutive et de la théorie du langage de Bühler $\gg 7$, mais aussi par rapport à la théorie de l'apprentissage et à l'utilisation de la perspective kantienne d'un point de vue hypothético-déductiviste.

La théorie hypothético-déductiviste de Popper est si connue que nous pourrions la croire évidente pour tous, mais il vaut peut-être la peine de rappeler ses traits essentiels de façon synthétique.

Ce qui conduit Popper à critiquer le néopositivisme est l'idée kantienne selon laquelle « notre intellect ne tire pas ses lois de la nature, mais impose ses lois à la nature ». La leçon que donne Kant à la tradition inductiviste réside dans le fait qu'il a montré qu'il n'existe ni observation pure, ni hypothèses, ni croyances, ni théories qui dérivent d'un stade de simple recueil des informations. Dans la théorie de Popper, l'apprentissage a lieu à partir d'un ensemble inné ou inconscient de réactions et d'expectatives, d'un mécanisme biologique en vertu duquel chaque animal est confrontéà son propre milieu ; les observations, elles, s'insèrent dans cet horizon inné d'expectatives, de réactions innées. Lorsque les observations font constater l'insuccès, l'échec des mécanismes de réaction innés, (et cela vaut pour les mythes préscientifiques comme pour les théories scientifiques), l'organisme (animal ou humain), produit de nouvelles solutions provisoires qui peuvent se présenter sous la forme de mutations organiques ou de nouvelles hypothèses. Chaque accroissement de connaissance consiste ainsi en la modification ou la correction de quelque phase de connaissance antérieure, " et donc, en dernière analyse, de quelque connaissance innée $»^{8}$. À ce stade, les observations servent à sélectionner des essais adaptés à la situation, parmi ceux produits librement par l'organisme. Decela découle l'hypothético-déductivisme de Popper ; à partir des hypothèses, on peut déduire de façon logique (sous la forme du modus tollens de la logique classique) des assertions empiriques de contrôle en mesure de corroborer ou de falsifier cette théorie. $M$ ais même lorsqu'elles s' avèrent corroborées, les théories scientifiques conservent un caractère hypothétique, parce qu'elles contiennent des généralisations qui ne peuvent être justifiées, qui ne peuvent être ramenées à des assertions singulières.

Pour en revenir au rapport avec Bühler, Popper, dans R eplies to my Critics, répond très significativement à Victor $\mathrm{K}$ raft à propos de la possibilité que le Cercle de Vienne, et en particulier D ie G rundformen der Wissenschaftlichen M ethoden (1925), aient influencé son anti-inductivisme. Cette œuvre, dans laquelle K raft développe une méthodologie de la science de matrice clairement déductiviste, s'avère être citée deux fois dans L ogik der Forschung. L'intéressé répond ainsi à la provocation de K raft : «En ce qui

7. Weimer, 1979, p. 286. Cf. également Donadon, 1987.

8. Popper, 1974a, p. 54. 
concerne les influences, je pense avoir été influencé bien plus par Karl Bühler, psychologue et représentant de l'école de Külpe, que par n'importe quel membre du Cercle ${ }^{9}$. Cette réponse, bien qu'extrêmement brève, presque fulgurante, est hautement révélatrice des influences - pour la plupart inexpliquées et restées dans l'ombre - de la formation de Popper, en ce qui concerne ce qui peut être considéré comme le véritable pivot de sa perspective. Concernant les années 1929-1930 (quand la rencontre avec $\mathrm{H}$ erbert Feigl lui fournit l'opportunité de publier ses idées sous la forme d'un livre), I'affirmation suivante mérite d'être soulignée : « Pendant cette période, j'avais I'habitude de penser que la critique queje faisais au Cercle de Vienne était le simple résultat de la lecture de Kant, et du fait que j'avais compris certains points particuliers de sa théorie ${ }^{10}$.

En mettant en relation les deux affirmations, on doit conclure, comme le suggère Bartley, qu'il y eut une corrélation effective entre l'activité et les réflexions psychologiques menées sous la direction de Bühler et I'utilisation de la perspective kantienne de façon déductiviste. Cependant, face à l'interprétation de Bartley, et surtout face à celle que nous pourrions appeler sa « received view », nous viennent deux considérations spontanées. La première, c'est que le fait de définir Karl Bühler comme un psychologue gestaltiste démontre que la connaissance de sa pensée n'est proportionnelle ni à sa célébrité ni à l'importance qu'on lui attribue. La seconde, c'est que l'on ne peut pas ne pas donner raison à Gaetano Kanizsa lorsqu'il affirme que, dans la $\mathrm{G}$ estaltpsychologie (par cette expression, on entend l'école de M ax Werthteimer), sont en vigueur certains « lieux communs courants », qui souvent se révèlent être imprécis, voire faux dans certains cas $^{11}$; le fait qu'il ait adhéré à des solutions de type kantien est précisément l'une de ces simplifications : en effet, le fait que les principes proposés par l'école de Wertheimer aillent dans une direction totalement opposée à ce qu'une telle affirmation devrait signifier (c'est-à-dire la reconnaissance de la fonction fondamentale de l'esprit dans l'organisation perceptive et théorique de la donnée) fut même l'un des arguments pour lesquels elle fut critiquée par de très éminents censeurs, Bühler en tête ${ }^{12}$. De ce point de vue, D ie beiden $G$ rundprobleme der Erkenntnistheorie - la version originale, qui, après des versions abrégées et les impitoyables coupures de Walter Schiff (I'oncle de Popper), parut sous le titre de Logik der Forschung - est un texte emblématique ; en effet, il permet de comprendre comment l'interprétation de K ant par Popper se place au sein d'une conception dont l'inspiration de fond se posait en net contraste avec les positions adoptées par la $\mathrm{G}$ estaltpsychologie de Wertheimer.

9. Popper, 1974b, p. 975-976.

10. Popper, 1974a, p. 86.

11. Kanizsa, 1984, p. XI.

12. O utre Bühler, cf. par exemple Goldstein, 1934, p. 328 ; M usatti, 1934, p. 8 ; Bruner et Postman, 1948, p. 17-118; Piaget, 1968, p. 84 et suivantes, et p. 99. 
Dans $\mathrm{D}$ ie beiden $\mathrm{G}$ rundprobleme der Erkenntnistheorie, bien plus que dans Logik der Forschung, c'est la solution d'un des thèmes charnières des réflexions du Cercle (l'indépendance de la théorie de la connaissance face à la psychologie de la connaissance, la nécessité de distinguer entre la façon dont naissent les théories scientifiques et leur validité), qui est fondamentalement mise en cause à travers le recours à la psychologie, recours, tient à expliquer Popper, qui ne signifie pas ouvrir la porte au psychologisme mais démasquer le « préjugé inductiviste » qui se cache derrière les positions néopositivistes.

Les prémisses qui donnent naissance au raisonnement de Popper sont aussi incontestables que fondamentales. La façon dont on découvre de nouvelles connaissances est une question de fait, et, par conséquent, nous sommes dans le domaine des jugements synthétiques. Contrairement aux jugements analytiques, ces derniers ne reposent pas sur le principe de contradiction, puisqu'un jugement synthétique peut être nié sans qu'on obtienne pour autant une proposition contradictoire : lenegatum d'un jugement synthétique est toujours logiquement possible ${ }^{13}$. Selon Popper, parmi certains représentants éminents du W einer K reis est présentel'idée quel'inductivisme représente l'unique explication possible relativement à la découverte de nouvelles connaissances (et donc relativement à une question de fait). L'explication d'une question de fait est donc transformée en quelque chose de "conceptuellement nécessaire » 14 . Popper affirme : « Si [...] I'on admet que dans la question relative à la découverte des connaissances, rien d'autre que ce qu'affirme l'inductivisme ne peut entrer en jeu, alors, par conséquent, on doit aussi admettre que dans la question mentionnée ci-dessus ce ne sont pas les simples faits qui décident ; ce sont les raisons logiques ou épistémo-logiques ${ }^{15}$. $M$ ais de cette façon, la confusion consécutive entre questions de fait et questions de valeur entraîne la disparition de la démarcation nécessaire entre théorie et psychologie de la connaissance (en substance, la distinction entre ce qui deviendra célèbre avec $\mathrm{H}$ ans Reichenbach sous le nom de « contexte de la découverte » et « contexte de la justification » des hypothèses scientifiques), et donc l'indépendance de la seconde par rapport à la première.

La cible polémique des critiques de Popper est sans doute D er logische A ufbau der Welt de Carnap, comme le confirme une affirmation significative contenue dans Conjectures and R efutations où Popper affirme, à propos de $D$ ie beiden $\mathrm{G}$ rundprobleme der Erkenntnistheorie: " $\mathrm{M}$ a critique s'adressait principalement à deux œuvres de C arnap, D er logische A ufbau der Welt, et Scheinprobleme in der Philosophie, mais également à certains de ses articles parus dans «Erkenntnis » ${ }^{16}$. N ous en arrivons donc à la cible des critiques de Popper : D er logische Aufbau der W elt de Carnap.

13. Popper, 1979, p. 13.

14. Ibid., p. 30.

15. Ibid., p. 21.

16. Popper, 1974c, p. 433. 
C'est bien connu, Carnap choisit comme base de son système le champ psychique propre, sous la forme du «solipsisme méthodologique ». À ce propos, il n'est pas de notre intérêt de nous arrêter sur le phénomène dit de l'A ufbau, ni sur les discussions qu'il a provoquées à l'intérieur du Cercle. $\mathrm{Ce}$ qui nous intéresse, c'est de montrer à partir de quels fondements et avec quels arguments $C$ arnap parvient, en partant d'une base subjectiviste, à la constitution d'une connaissance objective, universellement valide et intersubjective; c'est de montrer de quelle façon - selon ses propres paroles il résout le problème de savoir comment « atteindre l'objectivité de la connaissance, en adoptant également cette forme systématique », « éviter le danger du subjectivisme », et garantir l'indépendance du système de constitution de toute contamination possible à caractère psycho-logique ${ }^{17}$ : en somme, tous des objectifs que Popper partage entièrement, et auxquels il estime que le système de Carnap n'a pas satisfait, parce que ce système est entaché d'un préjugé inductiviste qui se reflète, de la psychologie de la connaissance, dans sa proposition épistémologique.

La classification qui caractérise la construction de Carnap est établie sur la « erkenntnismässige Primarität » des objets, c'est-à-dire sur le fait que d'un objet (qui, pour cette raison, est défini comme étant « cognitivement primaire ») résulte (logiquement et non psychologiquement) la condition nécessaire à la connaissance d'un autre objet (qui est appelé, pour cette raison, « cognitivement secondaire»). Par exemple, les objets physiques sont cognitivement secondaires par rapport aux objets psychiques, parce que les premiers se construisent sur la base des seconds; les objets de la psyché des autres sujets sont, à leur tour, cognitivement secondaires par rapport aux objets physiques, parce que la psyché des autres sujets est intelligible uniquement à travers des objets physiques, c'est-à-dire au moyen des mouvements expressifs, des réactions et des comportements des autres sujets.

C'est précisément en vertu de ce type de classification, parce que le système de constitution « exige» de tenir compte de «l'ordre cognitif des objets», de ce qui est « cognitivement primaire» et « cognitivement secondaire », que la base, le degré le plus bas du système de constitution, se situe dans les Erlebnisse, dans les données vécues, sous-entendues « dans leur totalité et dans leur unité de conscience ». Cela veut dire que l'on considère « le champ psychique propre » simplement comme on nous le donne, et non pas comme il devient par la suite dans «I'analyse psychologique»: Ies " atomes psychiques», tout comme les sensations, ne sont pas un fait primaire, mais des abstractions obtenues à partir de la donnée globale ; ils sont quel que chose de cognitivement secondaire. Ainsi, par exemple, dans la perspective adoptée par $M$ ach dans D ie Analysie der Empfindungen, « ce n'est pas la donnée elle-même qui est considérée comme l'élément fondamental,

17. Carnap, 1928, p. 181. 
mais les abstractions obtenues à partir de cette dernière, et donc quelque chose de cognitivement secondaire ». Par cela, Carnap se réfère à la psychologie de Cornélius et à l'école gestaltiste de K öhler et de Wertheimer, auteurs qui soulignent que « dans la perception, c'est l'impression gl obale qui est primaire, et que, à l'inverse, les sensations, les sentiments particuliers, etc. sont uniquement le résultat d'une analyse à caractère abstractif ». " L'accord, affirme Carnap, est primaire à l'égard du son particulier, l'impression du champ visuel global est primaire à l'égard des particularités qu'il renferme [...], les formes qui se trouvent dans le champ visuel sont primaires à l'égard des points colorés du champ visuel dont ces formes sont "composées" ${ }^{18}$.

La première conséquence fondamentale de cette option consiste en la difficulté qu'il y a à déterminer, dans les données vécues, une base solide et univoque non soumise au caractère arbitraire des contenus subjectifs, des courants individuels des vécus. U ne telle base, un tel point de départ du système de constitution (dans lequel on devra réduire les concepts scientifiques) est identifié par Carnap dans les « relations fondamentales » entre les vécus élémentaires, et ce, parce que si les vécus se trouvaient les uns à côté des autres sans relations, aucun progrès constitutionnel ne serait possible à partir de ces vécus : « Ce sont ces relations fondamentales, et non pas les éléments fondamentaux, qui forment les objets fondamentaux [...] dont sont constitués tous les autres objets du système. Du point de vue de la constitution, les relations fondamentales sont primaires par rapport aux éléments fondamentaux qui sont les membres de celles-ci ${ }^{19}$. À côté de cela, on trouve également l'idée que « la science est, par essence, science de la structure », et que tous ses objets de connaissance « ne sont pas contenu, mais forme ». La connaissance scientifique se sert en effet de descriptions de rapport (qui indiquent quel type de relations subsistent entre les objets), et non de descriptions de propriété (qui indiquent les qualités de chaque objet). Les descriptions de rapport et les descriptions de structure, c'est-à-dire l'ensemble des propriétés formelles des rapports qui existent entre les éléments d'un champ, permettent à Carnap de sortir de l'individualité des données vécues et du « danger du subjectivisme » auquel sa perspective semblait s'exposer à cause de la « base psychique propre » choisie comme fondement de tout le système ${ }^{20}$.

À la base de l'ordre objectal du système de Carnap se trouvent également la distinction entre « la val eur logique et la valeur cognitive » des assertions apportées et l'affirmation consécutive que chaque assertion qui entre dans le système subit nécessairement (pour des raisons logiques) une « transformation constitutionnelle » qui fait abstraction de sa valeur cognitive, psychologique et mentale; Carnap affirme qu'en ce qui concerne les rapports entre les objets, les assertions et les fonctions propositionnelles, « la méthode

18. Ibid., p. 156, 163-164, 183-184, 199.

19. Ibid., p. 182.

20. Ibid., p. 93-100, 181, 182. 
constitutionnelle [...] prend exclusivement en considération la valeur logique, et non pas la valeur cognitive ; une telle méthode est purement logique, et non pas psychologique ». Ainsi, dès le choix des relations fondamentales mises à la base du système, la « erkenntnismässige Primarität » s'applique au système de constitution exclusivement et ne doit pas être confondue avec « la valeur cognitive » des objets, qui se réfère à l'ordre psychologique concret. Le système de constitution fait abstraction du fait que, dans le processus cognitif, un rapport soit ou non fondamental ${ }^{21}$ : «II arrive, affirme Carnap, qu'un état de fait précis des choses soit certainement fondamental, du point de vuepsychologico-cognitif, et ne renvoie pas à des états de fait plus simples, mais que, logiquement, il dépende d'autres états, de façon à pouvoir être constitué par ceux-ci, faisant ainsi en sorte qu'il n'est pas nécessaire que précisément celuici soit considéré comme rapport fondamental $\gg^{22}$.

Tout en prenant acte des propos rassurants de Carnap, il s'agit cependant de voir de quelle façon il procède pour déterminer ces « propriétés structurelles » qui permettent de sortir du psychique propre et de garantir l'objectivité et l'intersubjectivité des formations du système.

Comme il s'agit d'unités non décomposables, Carnap adopte un instrument particulier pour le traitement des vécus et la description conséquente de leurs relations; il dérive du « principe d'abstraction » de R ussell. Parce que les données vécues se présentent justement comme des unités non décomposables, elles sont appelées " quasi-analyses », " quasi-caractéristiques » ou « quasi-ingrédients » se référant respectivement au procédé et aux formations que l'on obtient à partir d'un tel procédé. En second lieu, par rapport à l'« analyse authentique», la « quasi-analyse » d'un vécu n'invalide pas l'unité du vécu de départ, car elle l'insère dans une pluralité de connections d'affinité avec les autres « quasi-parts » d'autres vécus. De telles connections ou relations d'affinité peuvent consister soit en la concordance d'au moins un des quasi-ingrédients, (et donc en une égalité partielle), soit en une concordance seulement approximative d'au moins un des quasi-ingrédients (et donc en une similarité partielle). Ainsi se constituent des « cercles de similarité » en rapport avec de telles relations, et donc des classes, ainsi que des relations entre les classes ${ }^{23}$.

Cependant, les deux relations indiquées (d'égalité partielle et de similitude partielle) ne peuvent pas être considérées comme un fondement essentiel ; en effet, il existe une autre relation sur laquelle toutes deux reposent, une espèce de pierre angulaire, la clé de voûte de toutel'organisation du système de Carnap, sur la base de laquelle peuvent se constituer des cercles de similarité et donc des classes et des relations entre ces classes. Ce véritable pivot du système - qui complète, ou plutôt est à la base du cadre théorique

21. I bid., p. 182, 198 et 198-199.

22. Ibid., p. 158-159, 199.

23. Ibid., p. 186, 189, 192-195. 
que Popper prend pour cible - est appelé par Carnap le «souvenir de similarité ». Pour qu'à la fois la relation d' « égalité partielle » et celle de " similarité partielle » soient possibles, il est nécessaire qu'une « représentation souvenir » soit présente. Pour ces motifs, Carnap est convaincu que « les relations fondamentales » (ou « catégories » du système de constitution) ne peuvent logiquement dériver que d'une unique relation fondamentale. « Le nombre des catégories [...] est très restreint, et il n'y a probablement qu'une unique catégorie ». Une telle catégorie, une telle relation fondamentale est précisément le « souvenir de similarité ». Cependant, sur cette question, Carnap laisse échapper deux affirmations extrêmement significatives ; la première est que le fait que la similarité partielle découle du souvenir de similarité doit être compris comme « une constitution de forme non rigoureuse». La seconde est que le souvenir de similarité « est plus important également du point de vue cognitif ». M ais que signifient, dans ce contexte, « constitution de forme non rigoureuse » et « également du point de vue cognitif »? II est fondamental de se poser cette question, car à ce propos, on a la nette impression que C arnap remet en cause la « valeur cognitive » de la question, le point de vue psychologico-cognitif en plus du logico-cognitif. Pour déterminer une relation de similarité ou une relation d'égalité partielle entre deux vécus élémentaires $x$ et $y$, également du point de vue cognitif (psychologico-cognitif), il faut la « représentation souvenir » de ce qui précède les deux vécus, il faut que $x$ et y soient reliés à travers le souvenir de similarité : en somme, c'est cela que Carnap semble suggérer. $M$ ais en faisant cela, Carnap finit par accorder à ce qui n'est rien d'autre qu'une thèse psychologique une valeur incontestable; il finit par lui reconnaître un caractère indubitable égal à celui qui est le propre des vérités logiques : c'est-à-dire que par rapport à un phénomène psychologique bien précis (celui de la reconnaissance de ce qui est semblable), Carnap se réfère à un seul type d'explication, en tenant pour sûr qu'il n'y a pas d'alternatives ${ }^{24}$.

\section{3}

Cet entrecroisement symptomatique entre l'ordre logico-cognitif et l'ordre psychologico-cognitif n'échappe pas à Popper, qui voit dans cette véritable articulation du système de Carnap la proposition pure et simple d'une formulation psychologique tout à fait reconnaissable : « Elle admet que, en partant de chaque expérience vécue - et en particulier des expériences vécues de la perception - on parvient à nos connaissances, c'est-à-dire à nos expériences, à travers la généralisation de telles expériences vécues; que nous ordonnons les expériences vécues en fonction de leurs ressemblances [...], et nous obtenons ainsi les "cercles de ressemblance", et les "classes-abstractions" (Carnap). En procédant ainsi dans la direction inductive, du particulier au général, nous arrivons en dernier lieu aux concepts et aux connais-

24. Ibid., p. 201-204, 213. 
sances propres à la science. ${ }^{25}$. Popper est très clair lorsqu'il affirme que le foyer de sa critique ne porte pas sur l'inclination légitime pour une orientation psychologique aux dépens d'une autre. Par exemple, dans Theorie und Ehrfahrung in der Physik (1929), H erbert Feigl manifeste son penchant pour les raisons du déductivisme dans le cadre de la psychologie de la découverte tout en soutenant pourtant une perspective inductiviste en matière épistémologique. À ce propos, Popper n'est pas intéressé par l'idée de mettre en lumière le contraste de fond entre sa pensée et une solution de ce genre. $\mathrm{Ce}$ qui lui tient à cœur, c'est de montrer que la neutralité de la théo rie de la connaissance ne peut que passer par la reconnaissance de la légitimité du déductivisme, dans le cadre de la psychologie de la connaissance, par-delà le fait que, dans le domaine épistémologique (comme pour Feigl), on opte pour une perspective inductiviste. Le cas est différent en ce qui concerne Carnap, qui, selon Popper, est tellement conditionné par la tradition psychologique inductiviste qu'il la considère comme étant l'unique solution possible. Sous le couvert d'un langage délicieusement logique, ce qui n'est rien d'autre qu'une théorie concernant la psychologie de la découverte prend l'aspect de quelque chose de " conceptuellement nécessaire », de quelque chose qui n'admet pas $d^{\prime}$ alternatives ${ }^{26}$. De cette façon, la boucle est bouclée, mais ces conséquences vont miner tout le système de Carnap, car les deux pôles de la démarcation kantienne du quid facti et du quid juris se superposent littéralement. Une question de fait (celle de savoir si, dans la découverte de nouvelles connaissances, on utilise ou non des procédés inductivistes qui appartiennent à la psychologie de la découverte) est substantiel lement transformée en une question de valeur, annulant ainsi l'indépendance de la théorie de la connaissance face à la psychologie de la connaissance; ainsi, c'est le projet même d'une reconstruction rationnelle de la science qui vacille, celui d'une reconstruction indépendante du contexte de la découverte, dela manière dont on y parvient, de la manière dont on produit une nouvelle théorie, et de la question de savoir quels processus de pensée interviennent dans celle-ci ${ }^{27}$. Sans que $\mathrm{Car}$ nap en soit conscient, le préjugé inductiviste qui se trouve à la base de son système possède aussi un néfaste élément aggravant : il introduit l'épineux problème de l'induction, l'idée implicite - logiquement inadmissible d'une vérification inductive des théories, des assertions générales sur la réalité, à travers leur simple réduction à des propositions empiriques particulières ; et tout cela sans que Carnap ne mentionne jamais le problème de I'induction, avec cependant l'objectif de procéder à la constitution d'une épistémologie empiriste, bien sûr, mais également légitimée par un choix logique des concepts, des thèses, et des assertions utilisées.

25. Popper, 1979, p. 20.

26. Ibid., p. 30.

27. Ibid., p. 20-23. 
Pour rétablir un point de vue neutre et indépendant par rapport à la psychologie, pour combattre le préjugé inductiviste dont est entaché le système de Carnap, c'est justement à la psychologie qu'il faut avoir recours ; en effet, pour montrer que l'inductivisme psychologique n'est pas conceptuellement nécessaire, il faut montrer que le déductivisme psychologique est pensable et que, par conséquent, les thèses de l'inductivisme peuvent être niées sans que l'on obtienne comme résultat des propositions contradictoires, logiquement impossibles. Ensuite, ce sont les faits qui décident, affirme Popper, qui cependant ne manque pas de souligner que, selon lui, les faits « militent parfaitement bien » en faveur du déductivisme ${ }^{28}$.

Contrairement à ce qui transparaît dans le texte de Carnap, pour chaque solution proposée par l'inductivisme, des solutions de type déductiviste sont possibles et tout aussi légitimes. La reconnaissance de ce qui est commun à des situations différentes, comme nous l'avons déjà vu, est expliquée par l'inductivisme comme un cas classique d'apprentissage par la répétition : les réceptions de ce qui est semblable sont reliées par association, à travers le « souvenir de similarité ». À l'inverse, le déductivisme renverse les termes de la question en affirmant la suprématie génétique des expectatives, du bagage de réactions inné, de ce qui est « préformé subjectivement ». Dans ce cadre, la stimulation se présente comme « la condition matérielle de la réaction », dans le sens qu'elle détermine sa réalisation mais pas les « conditions formelles de son cours»: "Selon la conception déductiviste, nous ne parvenons pas à notre savoir empirique par abstraction ou par généralisation des perceptions sensibles; nous y parvenons en mettant à l'épreuve des anticipations qui sont provisoirement coordonnées au "matériel" des réceptions. C'est la valeur biologique qui décide si ces anticipations seront ou non abandonnées par la suite ». Sur la raison pour laquelle nous sommes portés à croire en des régularités ou en des lois de la nature, le contraste est analogue. Dans l'optique inductiviste, cette forme de croyance est déterminée par la répétition régulière de certains phénomènes : la répétition est donc en mesure de faire naître de nouvelles connaissances, en l'occurrence, la croyance que, dans la nature, il existe des régularités. Pour le déductivisme, à l'inverse, « la répétition ne génère rien $»$. L a recherche de régularité et l'attribution de régularitéà des phénomènes appartiennent au bagage inné des expectatives ; elles sont des réactions, des anticipations préformées subjectivement, avec l'aide desquelles nous " faisons "nos expériences" (mais dont nous ne sommes pas faits) »; elles ne sont pas obtenues à partir des réceptions, mais les précèdent. La répétition, et avec elle l'habitude qui en découle, sont en mesure d'accélérer, de perfectionner la mise à exécution de nos réactions, mais pas de les produire ${ }^{29}$.

28. Ibid., p. 21, 23.

29. Ibid., p. $26-27$ et 28-29. 
D e façon générale, le modèle de psychologie déductivisteque Popper a en tête, que nous pourrions appeler l'idéal type, est proposé par la perspective de Kant, et cela bien que le bilan général des solutions kantiennes soit, selon Popper, « insatisfaisant » pour une série de motifs bien connus : pour la façon dont il résout le problème de l'induction dans " I'A nalytique Transcendantale », pour avoir considérél'a priori commeétant nécessairement valideet vrai, indépendamment de l'expérience, et, enfin, pour «l'ambiguïté de l'expression a priori, du point de vue épistémologico-psychologique », pour cette confusion entre genèse et validité à laquel le K ant s'expose malgré lui dans K ritik den reinen Vernunft. Et cependant, malgré ces points de désaccord, Popper s'oppose «à ce qu'on dévalorise Kant, qui, aujourd'hui, recueille l'unanimité », et s'appliqueà défendre la pensée de $K$ ant, non seulement concernant sa méthode ( $c$ 'est-à-dire concernant la distinction entre quid facti et quid juri, et celle entre connaissance scientifique et connaissance non scientifique), mais concernant également « d'importantes parties des solutions qu'il propose »30.

$\mathrm{O} n$ a déjà souligné que la philosophie de Kant doit être considérée comme un facteur déterminant dans la définition du profil général de la formulation de Popper, en premier lieu concernant ce que l'on appellel'hypothético-déductivisme et la critique de l'idée selon laquelle l'observation devrait être considérée comme le fondement solide et indubitable, la suprême autorité, pour justifier les théories scientifiques. Dans l'optique de Popper, la grande leçon donnée par Kant à l'empirisme consiste précisément dans l'affirmation que la donnée, l'expérience, l'observation, sont toujours imprégnées d'expectatives et de théorie, qu'il n'existe ni pure observation, ni expérience passive, ni comportements dérivant d'associations faites passivement à partir des impressions ${ }^{31}$. M ais on sait tout aussi bien, comme cela apparaît dans tous les textes autobiographiques de Popper, que l'élan qui le poussa vers une telle formulation hypothético-déductiviste se dével oppa sur un terrain différent du terrain épistémologique ; en effet, il jaillit directement del'intérêt de Popper pour la psychologie de la découverte et pour les processus d'apprentissage. Et, en fait, dans $D$ ie beiden $G$ rund probleme der Erkenntnistheorie, Popper veut réé valuer, dans la perspective kantienne, «le point de vue déductiviste » opposé à celui, « inductiviste», du point de vue épistémologique et soutenu en particulier par Russell, Schlick, Wittgenstein et Carnap ; mais il veut surtout réévaluer cette psychologie de la connais-sance - « pour laquelle on n'a pas eu grande estime jusqu'à ce jour » - qui découle d'une lecture de type généticopsychologique de la perspective kantienne. Selon Popper, si l'on entend par a priori, non pas ce qui est «valide, indépendamment de toute expérience »,

30. Ibid., p. 19-20, 30-32. En ce qui concerne l'utilisation de l'expérience a priori par Kant, voir ibid., $\S 11$.

31. Popper, 1974a, 46-51, 61. O n peut aussi lire, dans D ie beiden G rundprobleme, « [...] la raison non dernière pour laquelle le positivisme échoue, c'est parce qu'il ne prête pas attention au concept kantien de connaissance » (Popper, 1979, p. 79). Cf., sur cet argument, ibid., p. 7678 et $\S 44$. 
mais ce « qui n'est pas généré sur la base de l'expérience », et si l'on comprend, de la même façon, la notion kantienne d'intellect comme «faculté des règles ${ }^{32}$, alors la loi de l'énergie spécifique des nerfs de J ohannes $M$ ühler (comme von $\mathrm{H}$ elmhotz l'avait déjà souligné), la perspective de l'école de W ürzburg d'O swald $\mathrm{K}$ ülpe (et en premier lieu celle réalisée par $\mathrm{K}$ arl Bühler et 0 tto Selz), et celle des « psychologues proches à la biologie », peuvent être considérées comme d'importantes « confirmations expérimentales». Parmi ces psychologues se distingue le nom d'Ernst $M$ ach ${ }^{33}$.

Sur la présence du nom de $M$ ach, une clarification s'impose. En effet, le fait qu'il soit présent dans cette brève liste peut surprendre, surtout si I'on se réfère au $M$ ach que l'on trouve dans les pages des néopositivistes, c'est-à-dire à l'image du $M$ ach sensualiste et empiriste, partisan d'une sémantique phénoménaliste des théories scientifiques, centrée sur les sensations, sur les données du sens ${ }^{34}$. Popper est parfaitement au fait de cette image, étant donné qu'il reproche fermement à $M$ ach sa vision de la conscience comme « pur et simple faisceau de sensations ${ }^{35}$. M ais cela n'empêche pas que Popper subisse profondément le charme de l'idée, développée par $M$ ach, du caractère évolutionniste, biologique, dela connaissance ; cela nel'empêche pas non plus d'opposer explicitement au $M$ ach sensualiste et inductiviste un $M$ ach déductiviste ${ }^{36}$, en mettant en lumière les aspects de sa pensée que l'histoire de la critique n'a soulignés que récemment avec l'emphase qui s'impo-sait ${ }^{37}$. En substance, il s'agit du $M$ ach (parallèle au $M$ ach sensualiste), qui accordeuneposition prééminente au système réactif, à l'ensemble des réactions propres à l'organisme (qu'il soit biologique ou psychique), dans les processus de la connaissance et de la pensée. De telles réactions (puisqu'elles sont innées) sont provoquées par des stimulations et des situations, mais leurs caractéristiques et leur progression dépendent du sujet, elles ne découlent pas de l'expérience. Parmi les différentes réactions dont dispose l'organisme, cellequi s'avère gagnante du point de vue biologique est coordonnée de façon stable à la stimulation, à la situation ambiante. Cette théorie occupeune position importante, surtout dans D ie Prinzipien der W ährmelehre. $\mathrm{H}$ istorisch-kritisch entwickelt, où c'est justement au sein de cette dynamique que $M$ ach détermine les « fondements psychologiques du concept » : concepts et hypothèses scientifiques sont donc une évolution, une transformation du savoir instinctif, dans l'effort constant que nous faisons pour résoudre les problèmes que l'environnement nous pose ${ }^{38}$. Selon Popper, nous nous trouvons clairement en face d'un modèle psychologique déducti-

32. Ibid., p. 20.

33. Ibid., p. 25, 30-31.

34. Pour avoir une idée de l'importance de cette interprétation de $M$ ach dans les années décisives de la formation du Cercle de Vienne, voir, par exemple, Frank, 1961, p. 21 et suivantes.

35. Popper, 1979, p. 65.

36. I bid., p. 503 note 6 .

37. Cf. par exemple Wolters, 1992, et Luccio, 1983.

38. M ach, 1896, p. 415, 422. II soutient une position similaire dans 1900 , chap. XIV. 
viste et « déjà au premier coup d'œil, on s'aperçoit qu'il contredit le sensualisme ${ }^{39}$ : I'affinité qui existe entre les idées de $M$ ach et la perspective hypothético-déductiviste de Popper est en fait considérable.

À présent quel'on a ferméla parenthèse concernant $M$ ach, il faut revenir aux considérations quej'ai esquissées dans les premières pages de mon exposé et se poser la question suivante : $D$ ans un des rares contextes où transparaît clairement l'importance de la formation psychologique de Popper, et où la critique du « préjugéinductiviste » de Carnap se développe précisément à travers le recours à la psychologie, pourquoi, parmi les psychologies partisanes de la perspective déductiviste, dont la formulation kantienne représente l'idéal type, la G estaltpsychologie (c'est-à-dire l'école berlinoise de Wertheimer) n'est pas citée au côté de noms tel que J. M ühler, Külpe, Selz, Bühler et M ach?

À mon avis, cette absence significative s'explique simplement par le fait que, chez Popper, la corrélation G estaltpsychologie-kantisme est totalement absente et que ce fut plutôt la pensée de Bühler, antagoniste déclaré de Wertheimer, W. Köhler, et $K$. K offka, qui contribua énormément à lui faire utiliser la perspective kantienne dans la direction indiquée.

Comme nous l'avons vu, Bartley soutenait, il y a plus de vingt ans, que la pensée de Popper devait « être comprise comme la pensée d'un psychologue gestaltiste, en premier lieu, maître d'école et néo-kantien ». L'ambiguïté et la simplification corrélationnelles de la définition de Popper comme « un psychologue gestaltiste » continuent lorsqu'on lit que Popper fut «façonné de façon décisive » par la pensée « de Bühler, de Külpe, et des autres psychologues gestaltistes ${ }^{40}$. Bartley parle, uniquement defaçon secondaire (et sans expliquer les raisons de la divergence), de discordances et de désaccords entre la perspective de Bühler, et la G estaltpsychologie de Wertheimer, mais cela ne suffit pas à clarifier la question de manière satisfaisante. Les versions encore plus simplistes de ce qu'a affirmé Bartley sont encore plus fausses ; dans ces versions, Bühler devient « un psychologue de la G estalt »41 tout court, ou encore, on peut lire que « sur le chemin qui porte au gestaltisme Popper "parvint à une position kantienne" ${ }^{42}$. Le défaut (et la confusion) de ces interprétations vient du fait que l'on parle de psychologues de la G estalt et de psychologues gestaltistes comme de quelque chose d'indifférencié. $M$ ais le fait de définir Bühler comme étant un psychologue de la G estalt est, dans le même temps, en partie juste et en partie profondément incorrect. C'est juste, car Bühler fut l'un des pionniers dans l'étude expérimentale des G estalten $^{43}$. Et c'est incorrect, car «psychologue de la G estalt » signifie trop de

39. Popper, 1979, p. 25.

40. Bartley, 1974, 13, 50-51; les mots ne sont pas en italique dans le texte original.

41. Corvi, 1993, p. 30.

42. Pera, 1981, p. 239. II faut préciser que Popper lui-même est en partie responsable du malentendu étant donné que, dans son Autobiographie, il définit Bühler comme « I'un des premiers psychologues de la G estalt » (Popper, 1974a, p. 30).

43. Cf. Bühler, 1913. 
choses, trop d'écoles de pensée profondément distantes entre elles, à tel point que Bühler fut en conflit constant avec la perspective de l'école de Wertheimer, c'est-à-dire la perspective que l'on désigne conventionnellement par l'expression $G$ estaltpsychologie. D'autre part, c'est la substance même de ce conflit qui montre que, alors que le binôme Bühler-kantisme est entièrement défendable, le binôme kantisme-G estaltpsychologie l'est beaucoup moins. À ce sujet, comme on parle de philoso phie autrichienne, on pourrait aussi bien parler d'une G estalttheorie autrichienne, qui comprendrait I'« école viennoise » de Bühler ${ }^{44}$, et l'école de Graz de Alexius M einong. La chose peut sembler paradoxale si I'on considère l'attitude fermée et méfiante réservée à la philosophie kantienne par la tradition autrichienne, mais ces deux écoles se rapprochèrent par les distances prises par rapport à la plus éminente version allemande de la G estalt (la Gestaltpsychologie de Berlin ou l'école de Wertheimer), et ce, pour se rapprocher de ce que devrait généralement vouloir dire être en harmonie avec la perspective kantienne : la mise en évidence du caractère actif et productif de l'esprit.

II serait long d'énumérer en détail tous les points de désaccord entre Bühler et les représentants de la G estaltpsychologie de Berlin (qu'il appelle « monistes structurels ») $)^{45}$. M ais s'arrêter, même brièvement, sur les caractères essentiels d'une telle discontinuité permettra de faire apparaître ce qui se cachait derrière la perspective de Bühler (et donc, à travers lui, derrière celle de Popper), c'est-à-dire les stimulations, les suggestions, et les solutions de l'école de W ürzburg d'O swald Külpe, dont Bühler fut (avant d'aller à Bonn, et donc à Vienne) un éminent représentant.

\section{4}

Dans l'interprétation de Bühler, par rapport à laquelle Popper se montra un fidèle disciple, la G estaltpsychologie était partisane d'un « nouveau physicalisme » car, à l'aide une conception purement « structurelle » de la stimulation et de la dynamique perceptive, et avec «I'aide d'un concept de structure défini de manière physicaliste », elle finissait par annuler le rôle du sujet et l'activité interprétative de ce dernier dans le processus perceptif. A insi dans l'optique de Bühler, toute la théorie du gestaltisme de Berlin gravite autour des conditions structurelles de la stimulation, des lois de l'organisation du champ perceptif (lois bien connues de ségrégation, de voisinage, de la bonne

44. Par « école viennoise de Bühler», on désigne les auteurs (dont les premiers sont $E$. Brunswik, L. Kardos, P.Lazarsfeld, et F. Kainz) qui gravitent autour du Psychologische Institut, dirigé par Bühler de 1922 à 1938. L'activité théorique et expérimentale de ces auteurs a été considérable; pour s'en faire une idée, on peut consulter la Z eitschrift für Psychologie de la fin des années 20, et l'A rchiv für di gesamte Psychologie du début des années 30. La mention « école viennoise de Bühler » a été utilisée sans grand succès dans la littérature spécialisée et est tombée en désuétude de façon prématurée, même si M usatti (c'est un cas plutôt isolé) l'utilise encore dans un article des années 50 (M usatti, 1953, p. 541). Dans la littérature contemporaine, M itchell Ash et Barry Smith I'ont, à juste titre, remise en circulation (cf. A sh, 1987 et 1988, et Smith, 1988, p. 229).

45. Bühler, 1927, p. 159, 132. 
forme, etc., énoncées pour la première fois par Wertheimer dans U ntersuchung zur L ehre der G estalt (1922)). De cette façon, la stimulation est comprise comme un ensemble de conditions qui, par le biais de sa propre configuration, conditionne la structure des processus physiologiques et, à travers ces derniers (sel on le principe gestaltiste de l'isomorphisme), la forme phénoménal $e^{46}$.

À l'isomorphisme psychophysique, Bühler oppose une hypothèse physiologique centrée entièrement sur l'activité nerveuse centrale, et donc, par le biais de cette dernière, sur le « moment central du contrôle », sur le rôle actif exercé par le sujet ${ }^{47}$. Par le biais d'une forte accentuation des aspects sémiotico-pragmatiques de la prestation perceptive (les données sensorielles sont des « indices » des propriétés des choses) ${ }^{48}$, Bühler propose ainsi une théorie de la perception entièrement centrée autour de l'activité interprétative du sujet qui perçoit et en contraste total avec une théorie (celle del'école de Wertheimer) qu'il considère commele « physicalisme dela marquela plus récente en psychologie», et dans laquelle il discerne « des affinités [...] avec le vieux spinozisme » et « la tendance à un réalisme aristotélicien et préaristotélicien totalement ingénu ${ }^{49}$. En synthétisant au maximum, on peut affirmer que la lecture de Bühler se présente, en tout point, en net contraste avec l'idée que la G estaltpsychologie a cherché « à démontrer que la théorisation, I'organisation [théorique], était une fonction fondamentale de l'esprit humain [...] » et donnait « la priorité à l'activité d'organisation de l'esprit [...] »50.

En fidèle disciple de Bühler, Popper adhéra totalement à cette interpré tation de la G estaltpsychologie, soutenant les raisons du maître et celles de son opposition à la formulation de l'école de Wertheimer dans certains ouvrages et critiques de textes à caractère psychologique et pédagogique, mais également dans sa recherche sur I'habilitation à enseigner ( $G$ ewohnheit und G esetzerlebnis, 1927), et dans Zur M ethodenfrage der D enkpsychologie, son mémoire de maîtrise soutenu en 1928. Dans cette recherche (dont Bühler fut le rapporteur, et Schlick, le corapporteur), Popper discerne une étroite similitude entre la perspective réductionniste soutenue par Schlick dans A llgemeine E rkenntnisl ehre et celle déterminée par la G estaltpsychologie, qu'il considère fondamentalement comme une exemplification de la première en milieu psychologique. Pendant ses années de pleine maturité, l'affinité qui existait avec la perspective de Bühler demeura évidente, à travers l'aversion que Popper montrait à l'égard de la solution isomorphiste proposée par Köhler en $1920^{51}$, de même qu'à travers sa proposition de la

46. Ibid., p. 87, 123-132. Cf. aussi Bühler, 1926.

47. Bühler, 1927, p. 128 et suivantes, p. 132.

48. Ibid., p. 109, 87-91.

49. Ibid., p. 129. Concernant la théorie physiologique de Bühler, voir Bühler, 1913, p. 291-297, Bühler, 1927, p. 124 et suivantes, Bühler , 1960, p. 53-59, 92 et suivantes, 120.

50. Bartley, 1974, p. 27-28.

51. Cf. Popper, 1977, p. 37-38. 
théorie (socratique et platonique) de l'esprit comme « mécanisme de contrôle », « comme capitaine d'un bateau ${ }^{52}$. M ais pour en revenir à $D$ ie beiden G rundprobleme der Erkenntnistheorie, d'où nous sommes parti, il faut souligner que c'est surtout sur l'interprétation de la notion kantienne d'«a priori » comme « anticipatif » de régularité que l'influence de Bühler se fait sentir. En effet, l'importance de l'interprétation bühlerienne du schématisme kantien, contenue dans Tatsachen und Probleme zur einer Psychologie der D enkvorgänge (1907), est évidente ; cette œuvre, rédigée pendant sa jeunesse et grâce à laquelle il obtint, à W ürzburg, sa Privatdozentur, représente I'une des meilleures œuvres qui ait été produites de toute la D enkpsychologie. Arrêtons-nous un instant sur la question du kantisme.

II est bien connu que la philosophie et la tradition kantienne furent accueillies en Autriche avec une méfiance et une hostilité évidentes (comme ce fut le cas, par exemple, pour Bolzano et la phénoménologie de Brentano), ou un désintérêt considérable. L'un des mérites historiques de Bühler, Viennois par adoption, lorsqu'il présenta les idées les plus fécondes de l'époque de Würzburg, a été d'avoir introduit, dans le contexte autrichien, une relecture de la perspective kantienne qui se révélait décidément plus proche de la sensibilité autrichienne car substantiellement réinterprétée à la lumière de la leçon de Brentano.

C'est en effet par le biais de l'école de W ürzburg que l'approche descriptive de l'étude des processus psychiques de la psychologie empirique de Brentano commence à être considérée comme faisant partie de la psychologie expérimentale au sens propre. La suggestion faite par Brentano concernait aussi bien la méthode que le sujet. En ce qui concerne la méthode, la perception intérieure, destinée à la description des actes psychiques et véritable pivot de la psychologie phénoménologique ou « psychognose » exposée par Brentano, est transformée en instrument scientifique et expérimental sous la forme de la révolutionnaire « introspection contrôlée » (une opération critiquée durement, cette fois, par Wundt et son école). En ce qui concerne les solutions théoriques, en particulier dans les œuvres et les réflexions de Bühler et d'O tto Selz (victime de la barbarie nazie à A uschwitz), cette version expérimentale de la perception intérieure fournissait un modèle général totalement similaire à celui de Brentano, c'est-à-dire centré autour de l'intentionnalité de la conscience, autour du fait que cette dernière ne se place pas passivement face au monde, mais s'adresse à lui de manière active, à travers ses propres actes et ses opérations. Chez Bühler, une partie importante de ce modèle actif de l'esprit est spécifiée précisément par le recours à la théorie kantienne du schématisme et au principe kantien selon lequel l'intellect doit être défini comme « la faculté des règles ». La partie la plus réussie et la plus intéressante des Tatsachen est en effet celle qui théorise la centralité de la « conscience d'une règle » (R egelbewußtsein) pour la solution des problè-

52. Ibid., p. 143, 132, 203. 
mes, mais aussi celle de la notion de schème en tant que règle utile à la structuration du contexte sensoriel et cognitif ${ }^{3}$. Dans le cas des problèmes cognitifs, qui sont au centre des Tatsachen, le schème s'objective à travers la conscience d'une méthode, qui sert à résoudre un ensemble précis de tâches : les éléments d'un champ sont donc organisés de manière corollaire à un tel schème. Concernant les concepts perceptifs, Bühler aura recours, dans son œuvre suivante, à la notion de schème pour expliquer les phénomènes de la constance perceptive lors du changement des conditions externes et internes. $D$ ans ces cas précis, c'est le schème qui nous permet de percevoir les éléments invariants des contenus sensoriels, d'en négliger les aspects secondaires, ce qui est contingent et imputable à la variation des conditions de l'environnement ; et c'est lui qui nous permet, au contraire, de mettre en valeur ce qui est discriminant, afin de reconnaître dans la donnée sensorielle un objet défini, une couleur définie, une forme définie ${ }^{54}$.

Comme le rappelle lui-même Popper, après Bühler, ce fut 0 tto Selz qui souligna que l'utilisation de schèmes se révélait fondamentale pour les opérations mentales mises à exécution par le sujet. Le schème permet d'interpréter une situation sensorielle ou cognitive problématique en l'intégrant, en la complétant, sur la based'une idée structurelle dont elle est justement une cohérence interne définie. Cette opération, que Selz appelle intégration d'ensembles (Complexergänzung), est, selon lui, à la base des processus de la connaissance et de la reconnaissance. La fonction d'un autre type de schème, le schème à caractère anticipant (das atizipierendes Schema), par lequel la conscience est comme guidée par un « savoir » anticipé, n'est pas différente. Grâce aux schèmes, se forment ainsi des « aptitudes de conscience » (Bewusstseinsanlagen), dans lesquelles est comprise la connaissance avec laquelle nous interprétons et faisons face aux situations ${ }^{55}$. D ans le cas de Selz également, l'étroite affinité avec l'hypothético-déductivisme de Popper est éclatante.

À ce stade, le moment est venu de faire le bilan. Dans $D$ ie beiden $G$ rundprobleme der Erkenntnistheorie, Popper rappelle, non sans sarcasme, I'hommage que $\mathrm{N}$ eurath rendit à Brentano pendant le premier congrès pour la science unifiée qui s'est tenu à Prague en 1929, pour « nous avoir épargné l'intermède kantien ${ }^{56}$. Dans $\mathrm{D}$ ie beiden $\mathrm{G}$ rundprobleme der Erkenntnistheorie, presque simultanément à la référence à $\mathrm{N}$ eurath qu'il vient de faire, Popper donne la preuve du rôle important qu'a joué Kant dans sa formation en lui offrant le terrain et les instruments à partir desquels critiquer le néopositivisme, et se sent dans l'obligation de « souligner de façon indélébile sa reconnaissance envers Kant ${ }^{57}$. Bühler propose, en Autriche, une relecture

53. Bühler, 1907, p. 339 et suivantes.

54. Bühler, 1927, p. 52-53, 95, et 1934, p. 305-308.

55. Selz, 1913, p. 314 et suivantes. Cf. également Selz, 1922.

56. Popper, 1979, p. 335 , et Neurath, 1930, p. 120 . "L'Autriche se soustrait à l'intermède kantien » deviendra également le titre du second chapitre de N eurath, 1935.

57. Popper, 1979, p. 336. 
de la perspective de Kant à la lumière de la leçon donnée par Brentano, la rendant ainsi plus agréable aux yeux d'une tradition (I'autrichienne), qui s'était toujours montrée profondément hostile envers Kant. Entre ces trois choses, je pense qu'il existe un lien qui a échappé à l'attention de la littérature critique ${ }^{58}$.

À la lumière de ce qui a été dit, je crois pouvoir affirmer ce qui suit : (1) il existe une grave lacune dans la reconstruction de la formation intellectuelle de Popper ; (2) cette lacune concerne une articulation fondamentale pour la compréhension des racines de sa perspective théorique; (3) cette lacune ne peut être comblée par la formule conventionnelle qui dit quePopper parvient à K ant par le chemin du gestaltisme ; (4) cette dernière formule s'avère aussi générale que fausse, et dangereusement réductrice.

C'est pourquoi, pour mieux comprendre les composantes et les stimulations qui ont joué dans la formation de Popper, on souhaite qu'une plus grande attention soit portée à la période de cette formation qui coïncide avec la seconde moitié des années 20 ( « années extrêmement importantes », nous dit Popper dans son A utobiographie). Un approfondissement de cette période balaierait certains malentendus sur la nature et les caractéristiques de la formation psychologique de Popper. Dans cette optique, le premier pas ne peut être que la publication de son mémoire de maîtrise et (comme cela a été le cas pour ceux qui furent publiés dans Erkenntnis) des articles et des textes critiques publiés entre 1925 et 1932 dans D ie Q uelle et dans Schulreform, les deux principales revues du mouvement de réforme scolaire d' $O$ tto Glöckel. Ces deux revues, nous dit Bartley, furent mises sous clé pendant la période de la censure nazie, mais, ajoute-t-il, elles sont aujourd'hui encore inaccessibles au grand public ${ }^{59}$.

\section{Bibliographie}

Ash, M.G., «Psychology and Politics in Interwar Vienna: the Vienna Psychological Institute, 1922-1942», dans Ash, M.C., Woodward, W.R., dir., Psychology in Twentieth-Century Thought and Society, Cambridge, Cambridge University Press, 1987, p. 143-164

58. L'influence exercée par Bühler pendant les seize années qu'il passa à Vienne fut décisive pour Popper, mais également pour d'autres auteurs. Parmi ceux-ci, on trouve bien sûr Wittgenstein, Egon Brunswik (représentant, après son émigration aux États-Unis, du mouvement pour la U nity of Science) et Konrad Lorenz. La philosophie du langage de Bühler et ses conséquences d'un point de vue perceptif furent marquants pour Wittgenstein (cf. M ulligan 1990 et 1997). En ce qui a trait à Brunswik, l'influence de Bühler concerna en particulier la notion d'intentionnalité (cf. Toccafondi, 1995, p. 243 et suivantes.). Enfin, eu égard à l'éthologie de Lorenz, il serait sûrement nécessaire d'approfondir l'influence des concepts de " déchaînement » (Auslösung) et de " relevance abstractive » développés par Bühler dans le cadre de sa théorie du langage (ibid., p. 250).

59. Bartley, 1974, p. 22. 
- - , « Die Entwicklung des Wiener Psychologischen Instituts 1922-1938 », dans Eschbach, A.E., dir., Karl Bühler's Theory of Language, A msterdam-Philadel phia, Benjamins, 1988, p. 303-325

Bartley III, W.W., "Theories of Language and Philosophy of Science as Instruments of Educational Reform: Wittgenstein and Popper as Austrian Schoolteachers », dans Cohen, R., Wartofsky, M., dir., Methodological and Historical Essays in the $\mathrm{Natural}$ and Social Sciences, Boston Studies in the Philosophy of Science, vol. XIV, Dordrecht, Reidel, 1974 (traduction italienne: Antiseri, D., L. Wittgenstein e K. Popper maestri di scuola elementare, Roma, Armando, 1976)

Bruner, J.S., Postman, L., «An Approach to Social Perception», dans Dennis, W., dir., Current Trends in Social Psychology, Pittsburg, University of Pittsburg Press, 1948, p. 17-118

Bühler, K., «Tatsachen und Probleme zu einer Psychologie der Denkvorgänge. I. Über Gedanken », Archiv für die gesamte Psychologie, 9, 1907, p. 297-365

- - , « Tatsachen und Probleme zu einer Psychologie der D enkvorgänge. II. Über G edankenzusammenhänge », A rchiv für die gesamte Psychologie, 12, 1908a, p. 1-23

- - , «Tatsachen und Probleme zu einer Psychologie der D enkvorgänge. III. Über Gedankenerinnerungen », Archiv für die gesamte Psychologie, 12,1908 b, p. 24-92

- - , Die Gestaltwahrnehmungen. Experimentelle Untersuchungen zur psychologischen und ästhetischen Analyse der Raum- und Z eitanschauung, Stuttgart, Spemann, 1913

- - , «Die "N eue Psychologie" Koffkas », Z eitschrift für Psychologie, 99, 1926, p. 145-159

- - , D ie K rise der Psychologie, Jena, Fischer, 1927 (traduction italienne de la $3^{\mathrm{e}}$ édition (Stuttgart, Fischer, 1965): Pusci, L., La crisi della psicologia, R oma, préface de R ohracher, H.,Armando, 1978)

- - , Sprachtheorie. Die Darstellungsfunktion der Sprache, Jena, Fischer, 1934 (traduction italienne de la $2^{\mathrm{e}}$ édition (Stuttgart, Fischer, 1965) : Cattaruzza Derossi, S., Teoria del linguaggio. La funzione rappresentativa del linguaggio, préface de F. Kainz,R oma, Armando, 1983)

- - , Das Gestaltprinzip im Leben des Menschen und der Tiere, BernStuttgart, H uber, 1960 (traduction italienne : Cattaruzza D erossi, S., II principio della Gestalt nella vita dell'uomo e degli animali, Roma, Armando, 1980)

Carnap, R, Der logische Aufbau der Welt, Hamburg, M einer, 1961 (traduction italienne: Severino, E., La costruzione logica del mondo, M ilano, Fabbri, 1966, p. 69-377) (édition originale : 1928)

Donadon, Cesarin E, «La teoria linguistica di Karl Bühler e la sua influenza sulla epistemologia di Karl Popper », Sapienza, 40, 1987, p. 431-434 
Corvi, R ., I nvito al pensiero di Popper, M ilano, M ursia, 1993

Frank, P., M oderne Science and Its Philosophy, N ew York, Collier Books, 1961 (traduction italienne: La scienza moderna e la sua filosofia, Bologna, II M ulino, 1973)

Goldstein, K., D er A ufbau der O rganismus. E inführung in die Biologie unter besonderer Berücksichtigung der Erfahrungen am kranken M enschen, The $\mathrm{H}$ ague, N ijhoff, 1934

Kanizsa, G., I fondamenti della psicologia della Gestalt, préface de W. M etzger, Firenze, Giunti Barbèra, 1984, p. IX-XX VIII

Luccio, R., «L'oggetto Interno del Sensista », in M elandri, E., dir., A nima ed E sattezza. L etteratura e Scienza nella Cultura A ustriaca tra ‘800 e ‘900, M arietti, Casale M onferrato, 1983, p. 156-165

M ach, E., D ie Prinzipien der Wärmelehre. H istorisch-kritisch entwickelt, Leipzig, Barth, 1900 (édition originale : 1896)

- - , D ie A nalyse der Empfindungen und das Verhältnis des Phychischen zum Psychischen, Jena, Fischer, 1900 (traduction italienne de l'édition de 1922 : Sosio, L., L'analisi delle sensazioni e il rapporto tra fisico e psichico, M ilano, Feltrinelli, 1975)

M ulligan, K., "Criteria and Indication », dans H aller, R., Brandl, J., dir., Wittgenstein - Eine Neubewertung, Akten des 14 Internationalen Wittgenstein-Symposiums, Wien, Verlag Hölder-Pichler-Tempsky, 1990, p. $94-105$

- - «The Essence of Language: Wittgenstein's Builders and Bühler's Bricks », Revue de M étaphysique et de M orale, 1997, p. 193-215

M usatti, C.L, « II costituirsi dell'esperienza come problema della psicologia empirica contemporanea », dans Atti del IX Congresso nazionale di filosofia, Padova, 1934

- - , « Ricerche sperimentali sopra la percezione cromatica », Archivio di Psicologia, N eurologia e Psichiatria, 14, 1953, p. 541-77

$\mathrm{N}$ eurath, 0 , W ege der wissenschaftlichen Weltauffassung, « Erkenntnis », 1, 1930

- - , Le développement du Cercle de Vienne et l'avenir de l'empirisme logique, Paris, 1986 (édition originale : 1935)

O Idroyd, D., The A rch of Knowledge. An Introductory Study of the History of the Philosophy and M ethodology of Science, $\mathrm{N}$ ew York and London, M ethuen, 1986 (traduction italienne: Di Libero Sosio, Storia della filosofia della scienza, M ilano, II Saggiatore, 1994)

Pera, M., 1981, Chi ha ucciso il positivismo logico?, dans Pera, M., dir., Popper e la scienza su palafitte, Roma-Bari, Laterza, 1982, p. 233-261

Piaget, J., Le structuralisme, Paris, Presses Universitaires de France, 1968 (traduction italienne: Bonomi, Andrea, Lo strutturalismo, M ilano, II Saggiatore Economici, 1994)

Popper, K. R., «A utobiography », dans Schilpp, P.A., dir., The Philosophy of Karl Popper, La Salle, O pen Court, 2. vol., 1974a (traduction ita- 
lienne: A ntiseri, D., L a ricerca non ha fine. A utobiografia intellettuale, Roma, Armando, 1978)

- - , « Replies to my Critics », dans Schilpp, P.A ., dir., The Philosophy of K. Popper, La Salle, O pen Court, 1974b, vol. II, p. 975-976

- - , « The Demarcation between Science and M etaphysics », dans Schilpp, P.A., dir., The Philosophy of K. Popper, La Salle, O pen Court, 1974C, 2 vol. ; aussi dans Conjectures and Refutations, London, Routledge and Kegan Paul, 1963 (traduction italienne: Pancaldi, G., Congetture e confutazioni, Bologna, II M ulino, 1972, p.431-498)

- - , Die beiden Grundprobleme der Erkenntnistheorie, Tübingen, M ohr, 1979 (traduction italienne: Trinchero, M., I due problemi fondamentali della teoria della conoscenza, M ilano, II Saggiatore, 1987)

Selz, O ., Ü ber die G esetze des geordneten D enkverlaufs, Eine experimentelle U ntersuchung, Stuttgart, Speman, 1913

- - , Zur Psychologie des produktiven Denkens und des Irrtums, Bonn, Cohen, 1922

Smith, B., « Gestalt Theory and Its Reception. An A nnotated Bibliography », in Smith, B., dir., Foundations of G estalt Theory, M ünchen-Wien, Philosophia, 1988, p. 227-478

Toccafondi, F., I linguaggi della psiche. Teorie della mente, della percezionee del comportamento da Würzburg a Vienna, M ilano, Guerini, 1995

Weimer, W.B., «La storia della psicologia e il suo recupero dalla storiografia », dans Caramelli, N ., dir., Storiografia delle scienze estoria della psicologia, Bologna, II M ulino, 1979, p. 271-304

Wettersten, J., Külpe, Bühler, Popper, dans Eschbach. A.E., dir., K arl Bühler's Theory of Language, Amsterdam-Philadelphia, Benjamins, 1988, p. 326-347

Wolters, G., "Ernst M ach e il "Verein Ernst M ach" », dans Haller, R., H empel, C.G., Wolters, G., von Wright, G.H ., dir., II Circolo di Vienna, Ricordi e riflessioni, Parma, Pratiche Editrice, 1992, p. 43-57 